ALPHA Nº 29 Diciembre 2009 (185-201)

ISSN 0716-4254

http://alpha.ulagos.cl

\title{
INTUICIÓN, LA EXPERIENCIA Y EL TIEMPO EN EL PENSAMIENTO DE BERGSON
}

Intuition, experience and time in the thinking of Bergson

Miguel Ruiz Stull*

Resumen

Esta indagación sobre la relación entre experiencia y tiempo en la obra de Henri Bergson tiene como propósito articular ambas nociones a partir de un análisis de la operación de la intuición en el contexto del pensamiento bergsoniano. De este modo, será posible considerar que el método de la intuición cobra un rol esencial en la emergencia de conceptos como duración, memoria y élan vital, los cuales colocan en relieve el singular empirismo con que puede ser ilustrado el discurso filosófico de Bergson.

Palabras clave: H. Bergson, experiencia, tiempo, intuición, metafísica.

Abstract

This article aims to investigate the relationship between experience and time in the philosophical work of Henri Bergson. Our purpose is to articulate these notions from an analysis of the operation of intuition in the context of this doctrine. This will likely consider that the method of Bergsonian intuition becomes an essential key in the emergence of concepts such as durée, memory and élan-vital, which highlight the singular empiricism with which Bergson's philosophical discourse can be illustrated.

Key words: H. Bergson, experience, time, intuition, metaphysics.

El proyecto filosófico de Bergson ${ }^{1}$ se caracteriza por la búsqueda de la precisión, noción que - en filosofía - no es o no coincide con una precisión de orden matemático. Para Bergson la precisión es un arte del matiz, es el emplazamiento de un poder recorrer las sinuosidades de lo real. Desde esta óptica, los sistemas filosóficos que no toman esto como imperativo devienen solamente en formales y vacíos. La empresa de la filosofía bergsoniana

\footnotetext{
${ }^{1}$ Emplearemos las siguientes siglas para las obras de Bergson aquí consideradas por nuestro estudio, cuya paginación corresponde a la edición original en francés de Oeuvres (1959): DI, Essai sur les données immédiates de la conscience (1889); EC, L'évolution créatrice (1907); PM La pensée et le mouvant (1934). Hemos cotejado estas versiones del original con las traducciones disponibles en castellano.
} 
podemos reconocerla por la emergencia de ciertos conceptos que resultan singulares dentro del devenir histórico de la filosofía. La novedad de la enunciación de conceptos como duración, memoria y élan vital sólo puede ser comprendida en su radicalidad siguiendo la forma o la configuración que cobra la experiencia desde un punto de vista filosófico. O bien, y desde otra perspectiva, la novedad radical de estas nociones pende necesariamente de la experiencia metódica de la intuición. En un sentido bergsoniano, la intuición no es una mera forma de acceso inmediato, una especie de sentimiento o de inspiración indeterminada: tanto la duración como la memoria no resistirían, en este sentido, ningún proceso que los defina en cuanto tales como conceptos. La intuición, para que sea considerada un método, debe esforzarse por volcarse sobre sí misma en un desinterés radical que ensanche las posibilidades de su objeto. La intuición, en este sentido, comporta la implicación en un acto simple de una multiplicidad dada por el objeto intuido ${ }^{2}$

Un esfuerzo de este género no es imposible; lo prueba la existencia en el hombre de una facultad estética al lado de la percepción normal. Nuestros ojos perciben los rasgos de un ser vivo, pero yuxtapuestos, no organizados entre sí. Se les escapa la intención de la vida; el sencillo movimiento que corre a través de las líneas uniéndolas y dándoles significado. En cambio, el artista trata de sorprender esta intención colocándose en el interior del objeto por una especie de simpatía y derribando — por un esfuerzo de intuición - la barrera que entre el modelo y él interpone el espacio. Cierto es que esta intuición estética, lo mismo hace la percepción exterior, sólo alcanza lo individual, pero cabe concebir una investigación orientada en el mismo sentido que el arte y que tome por objeto a la vida en general, al igual que la ciencia, siguiendo hasta el fin la dirección indicada por la percepción exterior y prolongando los hechos individuales en leyes generales. Indudablemente, tal filosofía nunca conseguirá un conocimiento de objeto comparable al que la ciencia consigue del suyo (2007:178).

Una precisión diferente es la que sitúa la pretensión filosófica bergsoniana. Para Bergson, la intuición siempre será un acto de simplicidad, pero lo simple no significa, en este contexto, la exclusión de "una

\footnotetext{
${ }^{2} \mathrm{Y}$ esto es así desde el comienzo en el trabajo emprendido por Bergson. Ya en 1944 se reconoce el uso de esta forma específica de aproximación a los problemas en filosofía. El carácter específico de esta apertura deviene en la enunciación consistente de los conceptos suministrados por el pensamiento de Bergson. Citamos, a título de ejemplo de nuestro punto de vista, este fragmento: "Cuando nosotros afirmamos que el número es uno, nosotros entendemos por ello que nos lo representamos en su totalidad por una intuición simple e indivisible del espíritu (intuition simple et indivisible de l'esprit): esta unidad contiene pues una multiplicidad, puesto que es la unidad de un conjunto” (1944):59-60.
} 
multiplicidad cualitativa y virtual de unas direcciones diversas en las que se actualiza” (Deleuze, 1987:10). Este punto — tomado por Deleuze en su comentario a Bergson - podría, en principio, abrir desde un nuevo lugar la discusión sobre la centralidad de la intuición en este proyecto filosófico. Sin lugar a dudas, es un punto notable, pero no es menos cierto que para Bergson el trabajo de la intuición está implicado en la diferencia y en la complementariedad que cobran la inteligencia y el instinto en el contexto de la vida. Desde esa importante página de Evolución creadora — que hemos citado- se hace razonable considerar una nueva apertura a la experiencia misma a la cual invita Bergson: una experiencia que aglutina un uso específico de la sensibilidad, por un lado, y una comprensión compleja de la realidad de un objeto que es la vida, por otro. Sensibilidad y vida entramados, sólo nos aparecen con precisión, esto es, en sus matices o variaciones, en la medida en que se efectúa este esfuerzo realizado por la intuición: el arte, en el ejemplo, nos provee la experiencia de lo individual y singular; la ciencia, por su parte, nos presenta su objeto en una generalidad en vistas de la acción orgánicofuncional sobre ello. En este horizonte, la filosofía debe ser capaz de darse su objeto en la complejidad y constante divergencia de su presentación, pero, a la vez, dar la regla de cómo esta multiplicidad, quizás virtual o posible, se actualiza en un momento y en un lugar determinados. Tal es el proyecto y la operación de la intuición que hace aparecer conceptos operativos como los de duración, memoria y élan vital que dan figura a una nueva forma de pensar una metafísica

La metafísica llegará entonces a ser la experiencia misma. La duración se revelará tal cual es: creación continua, fluctuación (jaillissement) ininterrumpida de novedad (1976:9)

Nuestro propósito se inscribe en esta línea antes esbozada. A saber, en vincular experiencia y temporalidad en el proceso mismo dado por el trabajo de la intuición, cuyo modelo siempre se nos ofrece de modo productivo y artístico: trama que se ve conjugada por una proyección de la metafísica fundada en una teoría de la experiencia. La intuición, entendida bajo el aspecto de un proceso de encuadre y distribución de lo sensible, siempre se nos aparecerá en el texto de Bergson, específicamente en Evolución creadora, no sólo anudada con el concepto de vida, sino que siempre se formulará a través de la distinción entre instinto e inteligencia, cosa que también se ilustra, como se observó preliminarmente, por el trabajo sobre la sensibilidad que realiza el artista. En otras palabras, si la filosofía de Bergson comporta un elemento creador — $\mathrm{o}$ si se quiere artístico- es, entonces, en la medida en que inflige una especie de presión a la sensibilidad en virtud de una 
construcción específica de su objeto. La intuición es un método y los conceptos son las sedimentaciones variables de su proceso, ya que deciden cada vez por recorrer aquello que Bergson llamaba las articulaciones de lo real, ${ }^{3}$ aquellos puntos en que la vida escoge una dirección que, en un principio, se ofrece como una multiplicidad virtual de direcciones por tomar. Si la intuición es el goce de la diferencia como lo estima Deleuze (2005:46-7) es porque realiza una integración de actos, plurales, en una actualización que, diferenciándose, muestra — en un aspecto - una articulación de su específica realidad, esto es, una línea de hechos que se extiende en la materia. En nuestra perspectiva, esto sólo es posible en la medida en que la experiencia se construya a partir de la temporalidad. Esto significa que las articulaciones de lo real se temporalizan, en cada ocasión, cuando estas se actualizan en una determinada línea de hechos (en definitiva, la duración para Bergson)

Lo que difiere por naturaleza no son las cosas, ni los estados de cosas, no son los caracteres sino las tendencias. Por ello la concepción de la diferencia específica resulta poco satisfactoria: no hay que atenerse a la presencia de los caracteres, sino su tendencia a desarrollarse (...). A lo largo de toda su obra, Bergson muestra que la tendencia no es solamente primera con respecto a su producto, sino con respecto a las causas de este en el tiempo, pues las causas se obtienen siempre, retroactivamente, a partir del producto mismo: una cosa, en sí misma y en su verdadera naturaleza, es la expresión de una tendencia más que el efecto de una causa (Deleuze, 2005:48).

Tendencia es una palabra decisiva en el contexto de la obra de Bergson, y Deleuze agudamente le presta la relevancia que posee. La naturaleza y la significación de la tendencia se identifican con el nombre de vida. La vida es tendencia, pero tendencia de actualización de una multiplicidad virtual, de series divergentes que inquieren por verse realizadas, por presentarse en su extensión en la materia. La tendencia es duración y, a su vez, la duración es vida. La vida, en este sentido, del mismo modo como se constituye la conciencia, ya de golpe instalada en la duración, crea algo

\footnotetext{
3 "Pero si se comienza por suprimir (écarter) los conceptos ya hechos, si se da una visión directa de lo real, si se subdivide entonces esta realidad, teniendo en cuenta sus articulaciones (articulations) los conceptos nuevos, que se deberán formar bien para expresarse, serán esta vez cortados a la exacta medida de su objeto (à l'exacte mesure de l'objet): la imprecisión no podrá nacer más que de su extensión con otros objetos que abarcarían igualmente en su generalidad, pero que deberán ser estudiados en sí mismos, fuera de estos conceptos, cuando a su vez se quiera conocerlos” (23).
} 
Intuición, la experiencia y el tiempo en el pensamiento de Bergson

En su bello libro sobre El genio en las artes, M. Séailles desarrolla la doble tesis de que el arte prolonga a la naturaleza y que la vida es creación. Gustosos aceptaríamos esta última fórmula; pero, ¿creación es, como el autor lo supone, una síntesis de elementos? Donde los elementos preexisten, la síntesis que se haga, estará virtualmente dada, ya que no es más que uno de sus arreglos posibles, arreglo que una inteligencia sobrehumana hubiera podido percibir de antemano entre todos los arreglos posibles. Creemos, por el contrario, que en los dominios de la vida, los elementos no gozan de existencia real y separada. Lo que hay son vistas múltiples del espíritu sobre un proceso indivisible. De ahí contingencia radical en el progreso, inconmensurabilidad entre lo que precede y lo que sigue, en una palabra: duración (1907:29-30)

Esta es una constante del estilo filosófico, o bien, de una forma de plantear el problema en Bergson: la referencia y insistente preferencia sobre la actividad del arte da una figura del proceso de producción de la vida. Pero esto es sólo una imagen del proceso, la evolución de la vida siempre implicará la articulación de una novedad, una imprevisibilidad y contingencia radicales liberadas, cada vez, de un programa o de un fin que preexista a su propio despliegue. Las articulaciones de lo real bajo esta perspectiva marcan una temporalidad, una duración que, diferenciándose, se hacen actuales en la línea de hechos: lo virtual, en un instante diferencial, se hace actual. En el horizonte de la vida, entonces, se hacen coincidentes tanto la experiencia como el tiempo, o bien, en otras palabras, la experiencia no puede reconocerse sino como tiempo: coincidencia que marca el proyecto de una metafísica futura en el contexto de Bergson. Las nociones de tiempo y de experiencia, implicadas con la operación de la intuición filosófica, nos informan de modo adecuado acerca de la serie de conceptos que identificamos como dominantes en el pensamiento de Bergson. O, según un punto de vista bergsoniano, la duración y la materia entran en juegos o encadenamientos cada vez variables respecto de la emergencia de sus propios conceptos. Un cuadro formado por los componentes variables de duración y de materia da lugar a los diversos aspectos involucrados en el problema, lo que no es sino entender las variaciones múltiples del fenómeno de la vida. Desde esta perspectiva, es posible sugerir que en Evolución creadora se asiste a la culminación, o enunciación definitiva, de aquello que aparecía latente, aunque concomitante, en sus trabajos anteriores, Datos inmediatos de la conciencia del año 1889 y Materia y memoria del año 1896, esto es, una metafísica de la vida, o bien empirismo radical, o bien simple vitalismo 


\section{Miguel Ruiz Stull}

Veremos que el problema del conocimiento, tomado de aquel modo coincide con el problema metafísico en que los dos dependen de la experiencia. En efecto, por un lado, si la inteligencia se dirige a la materia y la intuición a la vida, habrá que forzarlas (presser) ${ }^{4}$ mucho una contra la otra para extraerles la quintaesencia de su objeto; por lo tanto, la metafísica dependerá de la teoría del conocimiento. Por otro lado, si la conciencia se ha escindido en intuición e inteligencia es por la necesidad de aplicarse a la materia, a la vez, que de seguir el curso de la vida. El desdoblamiento de la conciencia dependerá, por tanto, de la forma doble de lo real y la teoría del conocimiento dependerá de la metafísica. En verdad, cada investigación conduce la una a la otra, forman un círculo que no puede tener sino por centro el estudio empírico de la evolución (1907-80).

Intuición, la experiencia y el tiempo, en el horizonte de la vida forman un cuadro que intercambian de modo variante sus perspectivas. En uno y otro caso, el concepto de metafísica es la tarea a desplegar por el esfuerzo filosófico bergsoniano. Sin embargo, la noción de metafísica que va formando, cada vez, Bergson implica o un esfuerzo de la intuición o un forzamiento de la sensibilidad. Suponemos que, en uno y otro caso, lo que se refiere es a una misma cosa: cómo o bajo qué condiciones es efectivo hacer aparecer la vida en cuanto tal. ${ }^{5}$ La vida, como hemos visto, coincide con la experiencia de la duración; pero, a la vez, la vida — al proceder por tentativas - requiere de la materia que constantemente le ofrece resistencia. Y esto es así, ya que

La vida manifestada por un organismo es un determinado esfuerzo para conseguir ciertas cosas de la materia bruta (...) inteligencia e instinto son dos formas de la actividad psíquica, y por sobre todo, dos métodos diversos de acción sobre la materia inerte (137).

\footnotetext{
${ }^{4}$ Quizá nuestra traducción pueda parecer arcaizante, sin embargo, da con el punto del que queremos dar cuenta: la intuición no puede ser entendida sin un reencuadre de la experiencia, una violencia que se ejerce a la sensibilidad, para darse su objeto de otra forma: "hay que hacer una violencia (brusquer) a las cosas, y por un acto de voluntad, empujar a la inteligencia fuera de sí misma" (195). El resultado de este proceso no es sino la intuición que "significa pues, por sobre todo, conciencia inmediata, visión que apenas se distingue del objeto visto, conocimiento que es contacto y hasta coincidencia (...) forzando (pressant) sobre los márgenes de un inconsciente que cede y resiste” (27).

${ }^{5}$ Bergson responde rápidamente a esto en virtud de aquello que a nosotros nos parece más problemático, a saber, la intuición: "Lo que nos llevaría al interior mismo de la vida sería la intuición, es decir, el instinto vuelto desinteresado, consciente de sí mismo, capaz de reflejar sobre un objeto y de ensancharlo indefinidamente” (178). Pero, inversamente, la inteligencia también guarda una relación con la intuición en la medida en que presenta la vida como un flujo indistinto, inanalizable ya que la inteligencia es un recurso privilegiado de mediaciones y, en consecuencia, de segmentaciones, en la materia (186).
} 
La materia siempre será, tanto para el instinto como para la inteligencia, un horizonte de acción, un lugar desde donde hay que sacar determinadas ventajas para proseguir con su curso. Esto es, ya, estar instalado en la duración, lo que coincide con el esfuerzo de la intuición, ya que entre instinto e inteligencia sólo existe una diferencia de grado, no de intensidad o de naturaleza. Instinto e inteligencia son dos tendencias - por igual, elegantes o económicas - de resolver los problemas que ofrece la materia. El problema de la diferenciación entre instinto e inteligencia sólo aparece por el uso de la intuición como método. Donde otros han visto una diferencia de naturaleza, ${ }^{6}$ para Bergson sólo es un mixto mal analizado, esto es, dos expresiones divergentes de una misma tendencia. La vida, según este aspecto, funciona a título de condición eficiente para que se produzcan o actualicen estos recursos para tratar con la materia

Si la fuerza inmanente de la vida (force immanente à la vie) hubiese sido ilimitada hubiera, quizás, desarrollado indefinidamente instinto e inteligencia en unos mismos organismos. Pero todo parece indicar que aquella fuerza es finita ${ }^{7}$ y que se agota muy pronto al manifestarse: que le es difícil avanzar mucho en varias direcciones a la vez, pues tiene que elegir y la elección que se le ofrece es entre dos modos de actuar sobre la materia bruta (142).

El vitalismo en la filosofía de Bergson se presenta como una potencia finita; una fuerza que se expresa como real en su elección, la cual se manifiesta, a su vez, de modo finito. Es, desde otro punto de vista, una serie divergente, múltiple y virtual que se actualiza en una dirección conveniente al progreso de la vida. Si el bergsonismo es una filosofía de la vida, lo es la razón de forzar las condiciones reales de la experiencia; forzar la sensibilidad para alcanzar la intuición de su objeto, de modo que pueda asistir con claridad $^{8}$ a las distinciones y matices que la constituyen. La metafísica

6 “El error capital que desde Aristóteles ha viciado la mayor parte de las filosofías de la naturaleza estriba en ver en la vida vegetativa, en la instintiva y en la racional tres grados sucesivos de una misma tendencia que se desarrolla, cuando son tres direcciones divergentes de una actividad que se ha dividido por el hecho de acrecentarse. Sus diferencias no son de intensidad ni más generalmente de grado, sino de naturaleza” (136).

7 Esta es la finitud que caracteriza al élan vital. Cfr. 127-142-150-254. Y no podría ser entendido de otro modo ya que el élan vital es duración, esto es, una virtualidad que aguarda por ser realizada cada vez y de ello se explica su constante divergencia: virtual y actual, ambos reales, se implican siempre en un proceso de constante, aunque variante, diferenciación. Cfr. Deleuze (2005:39).

${ }^{8}$ Claridad (clarté) tiene dos sentidos en Bergson: el primero, se expresa en la comprensión de lo nuevo a través de la medida de lo antiguo, función propia de la inteligencia que debe, en todos los casos, resolver los problemas que presenta la extensión de la materia. No obstante, y 
coincide con la experiencia en la medida en que es forzada por el acto simple de intuir que presenta las complejidades, quizá virtuales, del proceso de lo real, esto es, dirigidas hacia su serie efectiva o actual.

Los principales ingredientes de una filosofía de la vida que debemos considerar son, entonces, en primer lugar, que la diferencia vital ha de ser considerada como una diferencia interna en la cual la tendencia de cambio no es accidental, sino esencial; en segundo lugar, que las variaciones antes de ser consideradas como una simple combinación de relaciones de asociación y de suma de términos involucran instancias de disociación y de división; y finalmente, estas variaciones deben implicar una virtualidad actualizada que procede por vías de divergencia que siempre implican términos heterogéneos que se actualizan no en serie unidimensional, sino en una variación que cada vez es ramificada a partir de esta (Cfr. Ansell-Pearson 1999:67).

De esta forma, la vocación por la intuición aparece como un método de la hipótesis que articula y reúne, cada vez, lo que ahí se ofrece como un objeto vago e incomprensible. Las articulaciones de lo real distribuyen los elementos heterogéneos en lo que en la dimensión de los hechos aparece integrado: "Lo real es, al mismo tiempo, lo que se distribuye y lo que reúne" (Deleuze 2005:47). La tarea de la intuición consiste en esto: en considerar la claridad con que se da una novedad incomprensible, esto es, en no medirla con lo antiguo y, así, asimilarla en su duración para luego analizar esa complejidad que aclara otras complejidades. Según Bergson

Aceptémosla provisionalmente (...) la veremos, siendo oscura, disipar oscuridades. Por ella, unos problemas que juzgábamos insolubles van a resolverse, o más bien a disolverse, ya para desaparecer definitivamente, ya para plantearse de otro modo (31-2)

Tal es la tarea de la intuición que no es sino el proceso de integración variable de la intensidad que caracteriza a la duración, con su multiplicidad virtual, y a la materia que, ofreciendo problemas, inquiere por verse actualizada. Tras esto pareciese haber una lógica del asentimiento cuya data, creemos, no tiene nada de nuevo, ni siquiera para el propio Bergson, ${ }^{9}$ asentimiento

en un segundo sentido, "hay otra claridad que padecemos, y que se impone, por otra parte, a la larga. Es la idea radicalmente nueva y absolutamente simple, que capta más o menos la intuición” (31). La intuición, en efecto, aparece como un trabajo progresivo sobre lo simple de una idea que posee, virtualmente, vaguedades que inquieren por ser analizadas. "Pero, a falta del conocer, propiamente dicho, reservado a la inteligencia pura, la intuición podría alcanzar lo que hay de insuficiente en los datos de la inteligencia y dejarnos entrever la manera de completarlos” (178).

${ }^{9}$ Ante el asentimiento, pareciese sólo caber la anécdota como respuesta. Bergson dedicó sus primeros cursos a la filosofía epicúrea a través del estudio de Lucrecio (1883) reservando a la 
que opera al modo de una concesión lógica que tiene por fin hacer aparecer un objeto en la variación múltiple de sus expresiones. Desde esta perspectiva, duración y materia devienen en una fórmula más o menos abstracta que expresa los contenidos de la intuición, la emergencia y el desarrollo de conceptos originales que, cada vez, se entraman de modo distinto para dar cuenta en detalle, en todos sus matices, del problema planteado. Esbocemos, acá, un ejemplo desde una lectura esquemática del Ensayo sobre los datos inmediatos de la conciencia. A partir de los análisis a la psicología experimental de su época, Bergson insiste en que el tiempo, tomado de modo abstracto, no es sino un mixto o mezcla de espacio y de duración. Tomado, ya, sea desde la intuición o desde su proceso, esto significa que el espacio se presenta como una mezcla de materia y de duración y, ulteriormente, de materia y de memoria. Aquí se expresa este valor o diferencial de tendencia que sólo por vía de la intuición es posible enunciar: la materia es tendencia ya que se expresa como distensión; la duración — en cuanto es tendencia - se expresa como contracción. Aquí Deleuze, entre muchos otros que lo han seguido, ve el despliegue de la diferencia: la duración como virtualidad que presenta lo múltiple cada vez difiriendo de sí y la materia como actual que presenta lo uno que no difiere y, en consecuencia, se repite. ${ }^{10}$ Para nosotros, la

doctrina del clinamen la expresión de un asentimiento (Bergson 1937:24) y, posteriormente, a temas relativos a la filosofía de la naturaleza, especialmente la estoica y la noción fatum como producto de la misma extraña lógica (Bergson 2004:72ss.). En uno y otro sentido, hay una intención de despejar o desligarse del modelo aristotélico de pensamiento, posible de observar ya desde su tesis de habilitación, Quid Aristote de loco senserit (1889), gesto que fue subrayado, a su vez, por Burnet (1892) hacia el final de su reseña: esto es pensar que lo esencial es lo accidental, es decir, asentir sobre el trabajo progresivo de la formación de conceptos en filosofía, cuyo resultado más visible es la idea de duración. Tarea ciertamente por hacer y es, quizá, el lugar más especulativo del pensamiento de Bergson que concierne al rol de la fabulación como génesis radical del concepto en filosofía: fábula que seduce a la inteligencia en vistas de la unidad comprometida en el trabajo colectivo de la filosofía y su complementariedad divergente con la ciencia: "La ciencia eleva esta operación (la inteligencia) al grado más alto posible de exactitud y de precisión, pero no altera el carácter esencial. Como el conocimiento usual, la ciencia sólo conserva un aspecto de las cosas: la repetición. Si el todo es original, se complace en analizar en elementos o aspectos que a poca diferencia sean la reproducción del pasado. Y es que sólo puede obrar sobre lo que se supone capaz de repetirse, es decir, sobre lo que no se sustrae, hipotéticamente, a la acción de la duración. Se le escapa lo que haya de irreductible y de irreversible en los momentos sucesivos de una historia. Para representar esta irreductibilidad y esta irreversibilidad hay que romper con hábitos científicos que responden a exigencias fundamentales del pensamiento, efectuar una violencia (faire violence) al espíritu, hay que volver a subir la cuesta natural de la inteligencia y, esta es, precisamente la tarea de la filosofía” (29).

${ }^{10}$ Para un análisis detallado de las relaciones entre lo actual y lo virtual, del cual pende este argumento, sobre el que tendremos que volver a ello por lo extendido y lo profuso de su recepción en los últimos veinte años de discusión. Cfr. Deleuze (2005:51-3). 
intuición responde a un cuadro dinámico cuyos elementos son, efectivamente, la duración y la materia, pero que presentan el problema desde perspectivas diferentes: el signo, que efectúa los traspasos entre la aproximación y la presentación del problema como el proceso de disolución o replanteamiento, no es sino la claridad, o bien, constituir temporalmente la experiencia en un entorno situado en una noción fuerte de vida

Parece que la vida, en cuanto se contrajo en una especie determinada, perdió contacto con el resto de sí misma, salvo en uno o en dos puntos que interesaban a la nueva especie ¿Cómo no ver que en esto la vida procede como la conciencia en general, como la memoria? Arrastramos en nosotros, sin darnos cuenta, la totalidad de nuestro pasado; pero nuestra memoria no vierte en el presente más que los dos o tres recuerdos que han de completar por algún lado nuestra vida actual (168).

De esta forma contracción y distensión, una constante del texto bergsoniano, puede ser una manera adecuada de plantear, nuevamente, el problema de la subjetividad. ${ }^{11}$

El tiempo y la experiencia, la duración y la materia para Bergson constituyen, en nuestra hipótesis, un cuadro que cada vez sintetiza conceptos que lo componen. La vida, su concepto, se presenta como el lugar de la tentativa, pero que tiene lugar gracias al instante que la lleva a ser real. La clave contemporánea, emprendida desde su inicio por Deleuze, ha nombrado a esta operación como la realidad de lo virtual, el proceso de la diferencia donde lo virtual se hace actual. No podemos, en esta instancia, soslayar la fortuna que ha cobrado esta forma de plantear el problema.

\section{DIGRESIÓN: EXCURSO SOBRE LO VIRTUAL}

Una de las claves de lectura actuales para el pensamiento de Bergson, tomado ciertamente a partir de Deleuze como ya lo hemos indicado, ha insistido en brindar una positividad, una centralidad a la noción de virtual. No ignoramos ni desconocemos la relevancia de tan mentada noción; de hecho, la hemos tomado con cierta insistencia como término de definición ante una tentativa de lectura para la noción de intuición. Tampoco desestimamos

\footnotetext{
${ }^{11}$ La subjetividad no es más que la conciencia, lo que Bergson llama habitualmente vida interior: "una vida interior donde las fases sucesivas, tomadas cada una como única en su género, sean inconmensurables con el lenguaje” (1944:178). Lo dado como inmediato no es la diversidad sensible en la conciencia. Lo dado, en Bergson, es el flujo de la conciencia que es accesible a la inspección interior: una intimidad y complicidad del para-sí y el mundo. Cfr. Vieillard-Baron (2004:46-7).
} 
rápidamente la eficiencia interpretativa con que el concepto de virtual ha sido profusamente analizado en estos últimos años. ${ }^{12}$ No obstante, sostenemos que la noción de virtual corresponde a una instancia operativa — si se quiere metodológica- que daría cuenta o ilustraría en profundidad un vitalismo como tarea por emprender por el pensamiento filosófico, vitalismo que apuesta por una apertura ontológica que trasciende la operación misma que hace manifiesto su funcionamiento. La pareja actual/virtual, en este caso, ha de ser explicada en vistas de un proyecto mayor que bien podría tomar por nombre bergsonismo.

Nuestra lectura propone, habida cuenta de esta situación general en la exégesis de los textos, dar positividad a la noción de vida, de modo que (esbozado su concepto) sea posible interpretar cuál es la relación existente entre virtual y actual en vistas de su realidad. Desde Deleuze, al menos, se ha configurado un campo de distinción entre lo virtual y lo actual, ambos reales, para escapar de las categorías de lo real y lo posible, ${ }^{13}$ distinción que dominó con relativa amplitud el proyecto de una filosofía trascendental. Conforme a este nuevo plan, lo que se ha intentado es configurar un nuevo espacio para la experiencia y para su constitución, la cual ha cobrado como nombre genérico el de empirismo trascendental. Un empirismo radical que sostiene, a grandes rasgos, la exterioridad de las relaciones respecto de los términos vinculados, planteamiento que no se constituye, así, para una filosofía trascendental, en la medida en que estas relaciones penden necesariamente de la cosa, en cuanto fenómeno, y de su síntesis en cuanto tal, anulando así, en principio, su poder de variación. ${ }^{14}$ Más allá de estas consideraciones generales que, de suyo,

\footnotetext{
${ }^{12}$ Para estos numerosos trabajos remito a las referencias de este ensayo. Sólo cabe destacar un solo disenso respecto de la mentada positividad tanto de las fuentes como de sus interpretaciones en el trabajo de Mullarkey (2004:479ss) quien sostiene un retorno al programa de un psicologismo, constituido por la precisión de las improntas que en el ánimo dejarían lo real. Sostenemos que esta interpretación no es consistente, al menos, desde el propio Bergson, en la medida en que, justamente, lo virtual se aloja en su potencia de actualización, en cuanto juego de la memoria y la duración, juego que da pie a la emoción que, cada vez, selecciona un contenido de recuerdo, juntura que hace indiscernible, al menos en este punto crítico, la distinción entre cuerpo y alma en el continuo del movimiento de contracción (Vida) y distensión (Materia) como es presentado por Bergson a lo largo de Evolución creadora.

${ }^{13}$ Es ya célebre esta enunciación dada por Deleuze, fórmula que es retomada en muchos lugares de su obra, que bien puede valer como articuladora de su propio pensamiento: "Desde un determinado punto de vista lo posible es lo contrario de lo real, se opone a lo real; pero también lo virtual se opone a lo actual, lo cual es algo completamente distinto (...) lo posible no tiene realidad (aunque pueda tener una actualidad); inversamente, lo virtual no es actual, pero posee en cuanto tal una realidad”. Deleuze (1987:102).

${ }^{14}$ Todos los desarrollos y formulaciones de esta cuestión han tenido su fuente desde Empirismo y subjetividad, retomados por Buchanan (1999:104-5) Martin, en Buchanan (1999:246-7) por citar los estudios más ampliamente referidos.
} 


\section{Miguel Ruiz Stull}

constituyen ya un problema, ${ }^{15}$ creemos que la noción de empirismo como tal posee una relevante función en la medida en que pueda ser lícita una formulación que vaya más allá de una simple forma de constitución de conocimiento, de modo que defina el núcleo de una actividad práctica que puede o no puede tener en vistas la adquisición y dominio de tal o cual objeto que aparezca en el contexto de la experiencia. ${ }^{16}$ El empirismo, en este caso, designaría un coeficiente diferencial de la sensibilidad, tomando muy en serio las variantes de las relaciones; ${ }^{17}$ esto puede ser tomado como lo virtual, ${ }^{18}$ tanto en

${ }^{15}$ Problema cuya formulación, en Deleuze, puede ser brevemente presentada a partir de un importante texto de Empirismo y subjetividad de Bergson: "La crítica es empírica cuando, situándose en un punto de vista puramente inmanente desde el cual sea posible una descripción que halla su regla en hipótesis determinables y su modelo en física, uno se pregunta a propósito del sujeto: ¿Cómo se constituye en lo dado? La construcción de este cede su lugar a la constitución de aquel. Lo dado ya no está dado a un sujeto; el sujeto se constituye en lo dado” (2002:93). Y, en este contexto, lo dado no es más que ese flujo y colección de impresiones separables y distinguibles $\mathrm{y}$, en consecuencia, diferentes. Y esta diferencia que provee la experiencia entendida como sucesión, movimiento y cambio, se vuelve en principio regulador de la distinción y separación de ese flujo. El sujeto, en este contexto, se constituye en lo dado porque cree e inventa: "creer es inferir de una parte de la naturaleza otra parte, no dada en este caso. E inventar es distinguir poderes, es constituir totalidades funcionales, totalidades que tampoco están dadas en la naturaleza” (Deleuze, 2002:92). Este punto o diferencia, como hemos visto, distingue a la inteligencia, que mide desde lo antiguo; de la intuición, que se propone desde lo nuevo como poder de analizar y componer, cada vez, el aspecto de lo real en sus fluctuaciones o articulaciones. Con esta noción de fluctuación o fluxión Bergson - detectada por Alliez (2002) desde Newton- “alcanzará los conceptos fluidos, capaces de seguir la realidad en todas sus sinuosidades y de adoptar el movimiento mismo de la vida interior de las cosas” (Alliez, 2002:105). Esto no es sino investir a la metafísica con el cálculo infinitesimal, introduciendo la variable tiempo, de un movimiento constituyente y diferenciante tanto en el ser como en el pensamiento. Según Alliez, esto corresponde nombrarlo a título de una ontología de lo virtual.

${ }^{16} \mathrm{Y}$ así es propuesto por Bergson (1987) en una página decisiva de El pensamiento y lo moviente: "Un empirismo verdadero es aquel que se propone estrechar tanto como sea posible el original mismo, profundizar la vida y, por una especie de auscultación espiritual, sentir palpitar el alma; y este empirismo verdadero es la verdadera metafísica (...) empirismo que no trabaje más que a medida, se ve obligado a suministrar un esfuerzo completamente nuevo para cada nuevo objeto que estudia” (1987:134-5). El subrayado es nuestro.

${ }^{17}$ También lo plantea Yankelevitch (1962) respecto de la naturaleza reductora de los conceptos, cuando señala que: "La ilusión vulgar descansa en el prejuicio de que las cosas se fabrican discursivamente y a trazos tal como se dejan analizar actualmente bajo nuestros ojos, pero en el orden inverso; la síntesis por la cual son construidas reproduciría, invirtiendo el orden, los grados sucesivos que atraviesa el análisis para descomponer su forma en elementos” (1962:181). Punto kantiano expresado por Yankelevitch que remite, creemos, a una lectura complementaria de las “Deducciones trascendentales A y B” de Crítica de la razón pura. Cfr. Khandker, 2004. En nuestra hipótesis, dejamos en suspenso esta posibilidad, aunque no disminuimos una relevancia ulterior frente al problema de la constitución de la experiencia, sobre todo observando la estrategia de Deleuze que enlaza la intuición bergsoniana con la filosofía postkantiana en Diferencia y repetición (2002a). Cfr. Alliez (2002:105). Un buen 
Bergson como en Deleuze, a partir de una posible lectura al empirismo de Hume. ${ }^{19}$ De esta forma, pensar en virtud de un principio constitutivo dado en la categoría de relación como diferencial de las asociaciones esbozaría una nueva imagen del sujeto de la experiencia. Inversamente, esto es, que el pensamiento tendrá por objeto al pensamiento mismo ${ }^{20}$ en el contexto de la vida. En efecto, este rasgo que ha dado tantos rendimientos interpretativos para las lecturas de Deleuze es un rasgo primordialmente bergsoniano, al menos desde la aparición de Materia y memoria de $1896^{21}$ donde se vinculan

estudio sobre la relación entre Bergson y la intuición respecto de su correspondencia con Kant ha sido presentado por Moulard-Leonard (2006) indicando una cierta familiaridad, que nos parece útil y precisa, entre la noción ampliada de trascendental kantiana con la idea de virtualidad, sobre todo atendiendo al segmento de la deducción del juicio de gusto en Crítica a la facultad de juzgar. Cfr. Moulard-Leonard (2006:148-9).

${ }^{18}$ Como lo señala Deleuze: "Lo propio de lo virtual es existir de tal forma que solo se actualiza diferenciándose, que se ve forzado a diferenciarse, a crear líneas de diferenciación para actualizarse" (1987:102).

19 Deleuze, en un artículo de 1956 referido a Bergson, señala esta mentada afinidad: "El proyecto que hallamos en Bergson, el de alcanzar las cosas rompiendo con las filosofías críticas, no era absolutamente nuevo, ni siquiera en Francia, ya que define una concepción general de la filosofía que, en muchos aspectos, participa del empirismo inglés” (2005:43). Dicha concepción, puesto acá en términos en sumo esquemáticos por Deleuze, opera en dos niveles en Bergson: primero, en la tentativa de determinar las diferencias de naturaleza entre las cosas, captarlas en su ser y alteración y, segundo, si esto es así, es posible considerar que la misma diferencia sea algo, que posea alguna naturaleza y que nos presente de algún modo el ser. Los planos metodológico y ontológico, respectivamente, remiten, así, al análisis de las diferencias de naturaleza y la determinación de la naturaleza de la diferencia, esto es, la intuición como aproximación desde la duración a la materia. Para una formulación respecto de Hume, donde intuición bergsoniana y asocianismo empirista se encuentran. Cfr. Deleuze (2002b:112-3).

${ }^{20} \mathrm{O}$ bien hacer pensable lo impensado en el pensamiento bajo el modelo de hacer visible el aspecto invisible de lo visible: en el fondo, crear las condiciones de una nueva sensibilidad, condiciones que remitirían a la parte de trascendental que se aloja en el proyecto de este empirismo. Cfr. Martin (1993:229ss).

${ }^{21}$ Esto podría ser formulado de la siguiente manera: si en Ensayo sobre los datos inmediatos de la conciencia se efectúa sobre la distinción problemática entre espacio y duración, la cual reenvía al problema metafísico del dualismo, se sigue en Materia y memoria que el problema se reconsidera sobre un plano psicológico fundado en la idea de duración de carácter metafísico que permitiría en principio unificarla en la unidad de la materia. Cfr. Worms (2004:16-7 y, específicamente, 151-4). El problema de esta nueva dualidad se verá problematizado respecto de la noción de vida, que aquí nos ha ocupado, la cual se definirá por su esfuerzo de resistencia respecto de la materia, alcanzando así, en la Evolución creadora, la diferencia de naturaleza que en las obras anteriores sólo se manifestaba en diferencias de grado, proceso conceptual dado por el trabajo de la intuición: recobrar el dualismo en la integración de una naturaleza, de la naturaleza de la diferencia, "si el élan vital se diferencia a sí mismo, es porque la materia está en oposición a este impulso; ahora vemos que diferenciación tiene su origen en la interioridad de la duración con ella misma” (Deleuze, 2007:90). 
íntimamente las nociones de tiempo, materia y vida. Respecto de la vida, tanto la duración como la memoria se conjugan en la experiencia singular dada por el proceso de la intuición: proceso que da luces de la relación entre duración y materia.

Este arte de las distinciones, como puede ser entendida la intuición y que caracteriza a Bergson, según la visión de Deleuze, sólo puede manifestarse en sus problemas y en sus resoluciones bajo la constancia de un núcleo o de un punto de diferenciación: el lugar donde la contracción asiste a su despliegue. Muchas dicotomías visitadas por Bergson en Evolución creadora (como vegetal/animal, instinto/inteligencia) son falsos mixtos, ya que cada uno opera en su serie como soluciones de un mismo problema, lo que no es más que la resistencia que la vida ofrece frente a la materia: el despliegue de un mismo impulso que se distribuye, en cada caso, entre líneas de desarrollo divergentes: he ahí la naturaleza de lo virtual que se impulsa en una actualización dada. ${ }^{22}$

\section{CIERRE: LA VIDA COMO ENCLAVE DE TIEMPO Y EXPERIENCIA EN EL PROCESO DE LA INTUICIÓN}

La vida es la serie de tentativas que se traducen en divergentes series específicas que resisten la materia. En este sentido, la materia siempre se comportará como un obstáculo, como un problema que alienta diversas, pero igualmente efectivas, formas de resolución: la contracción de estas no es sino duración. La materia es siempre lo que se distiende y la vida lo que siempre se contrae en sus diversas formas por este trabajo o esfuerzo sobre la resistencia de la materia. Este lugar, como esbozamos, viene dado por el complejo duración-materia, núcleo del pensamiento de Bergson, en virtud de los conceptos que produce; a saber, una nueva imagen de la memoria y de la vida que no es sino pensar desde otro punto la configuración de la experiencia, lo que no es sino una definición tentativa de intuición. O bien, la serie de conceptos - que al inicio consignamos como característicos del pensamiento de Bergson - pueden ser entramados por el método de la intuición siguiendo el siguiente esquema: la duración es la diferencia que, cada vez, difiere de sí misma funcionando como principio de determinación; la memoria se concentra en la coexistencia de grados de diferencia cobrando el valor de una determina-

\footnotetext{
${ }^{22}$ Por ejemplo, como es posible de ser dicho a propósito de la inteligencia y el instinto: "Puesto que el instinto no existe más que en un estado de huella, o de virtualidad, puesto que no es lo suficientemente fuerte para provocar actos o para impedirlos, deberá suscitar una percepción ilusoria o por lo menos una imitación de recuerdo bastante precisa, bastante sorprendente para que la inteligencia se decida por ella” (95).
} 
ción recíproca y, finalmente, el impulso vital es el proceso de diferenciación de la diferencia como principio de determinación que integra el objeto en conformidad con su concepto. ${ }^{23}$ Tal es la intuición: tallar un concepto apropiado a su objeto moldeado por las fluctuaciones de lo real, un "concepto del que apenas puede ser dicho que sea un concepto” (197).

De esta forma, Bergson ofrece una definición de los movimientos de lo viviente conforme a esta especie de suspensión temporal que proporciona la memoria en cuanto a duración. Es así como la vida se contrae, o bien, (a) tiende su acción en la duración sobre la materia que siempre ha de ofrecer resistencia. Así como las plantas, en una función dada por la clorofila, acumula energía fijando nitrógeno o carbono, o bien, como los animales se desarrollan orgánicamente bajo la forma de instinto o inteligencia, es posible comprender la vida desde este punto axial que explota lo que ahí virtualmente se ofrece a la acción. De esta manera, el impulso vital —más que desenvolverse por una especie de espontaneidad - marca este valor de tendencia que se exterioriza en virtud del dominio de la materia a la vez que permite comprender la vida a partir del desarrollo de una intuición, intuición correlativa al método de saber que practica Bergson. En suma, el movimiento de contracción que efectúa la vida pone en evidencia este espacio de lo virtual, espacio ciertamente inextenso, ya que sólo fija su valor en la medida en que se hace exterior, que actualiza una articulación de lo real en una línea de hechos por divergencia o por diferenciación. Clinamen o libertad, nociones revisadas por el propio Bergson en el proceso de su pensamiento, podrían ser sinónimos de este impulso en que se desenvuelve la vida, sólo en virtud de que una y otra noción pongan en relieve su aspecto físico o material de un hacerdetonar; un explosivo que hace desplegar movimientos cada vez más fuertes en sus efectos de realidad.

\section{Universidad Diego Portales* Instituto de Humanidades Ejército 278 / 837005, Santiago (Chile) ruizstull@gmail.com}

\footnotetext{
${ }^{23}$ Para la relación entre imaginación y pensamiento metafísico, se puede ver en una aguda inspección realizada por Miquel (2007:99ss.).
} 


\section{BIBLIOGRAFÍA}

ALLIEZ, Eric. "Sobre el bergsonismo de Deleuze”, en E. Alliez (Ed.). Gilles Deleuze. Una vida filosófica. Medellín: Euphorion, 2002.

ANSELL-PEARSON, Keith. Germinal Life. NY, Routledge, 1999.

BERGSON, Henri. La evolución creadora. Buenos Aires: Cactus, 2007.

------ "The metaphysics of Life”, en Substance 36.3 (2007):25-32.

------ Essai sur les données immédiates de la conscience. Paris: Critique de F. Worms, PUF, 2007.

------ L'évolution créatrice. Critique de F. Worms. Paris: PUF, 2007.

------ Leçons clermontoises I y II. Ed. R. Ragghianti. Paris: L’Harttman, 2004.

------- El pensamiento y lo moviente. Madrid: Espasa-Calpe, 1976.

------ Oeuvres. Paris: PUF, 1959.

------- Ensayo sobre los datos inmediatos de la conciencia. Montevideo: García \& Cía, 1944.

------ Lucrecio. Montevideo: Hiperion, 1937.

------- La evolución creadora. Madrid: Nacimiento, 1912.

BUCHANAN, Ian. A Deleuzian Century? Durham: Duke UP, 2005.

BURNET, John. "Review: Aristotle's Theory of Topos”, en The Classical Review 6.7 (1892): 322.

DELEUZE, Gilles. "Lecture Course on Chapter Three of Bergson's Creative Evolution”, en Substance 36.3 (2007):72-90.

------ La isla desierta y otros textos. Valencia: Pre-textos, 2005.

------- “Un retour à Bergson”, en Deux Régimes de Fous. Paris: Les Édtions de Minuit, 2003.

------- Empirismo y subjetividad. Barcelona: Gedisa, 2002b.

------- Diferencia y repetición. Buenos Aires: Amorrortu, 2002a.

------ El bergsonismo. Madrid: Cátedra, 1987.

GUNTER, Pete A. Y. "Bergson's Creation of the Possible”, en Substance 36.3 (2007):33-41.

KHANDKER, Wahida. "Bergson, Kant, and the Evolution of Metaphysics”, en Pli 15 (2004):103-24.

MARTIN, Jean-Clet. Variations. Paris: Éditions Payot \& Rivages, 1993.

MIQUEL, Paul-Antoine. Bergson ou l'imagination métaphysique. Paris: Kimé, 2007.

MOULARD-LEONARD, Valentine. "The sublime and the intellectual effort: The imagination in Bergson and Kant”, en Journal of British Society of Phenomenology 37.2 (2006):138-51. 
Intuición, la experiencia y el tiempo en el pensamiento de Bergson

MULLARKEY, John. "Forget the virtual: Bergson, actualism, and the refraction of reality", en Continental Philosophy Review 37. Netherland: Springer (2004):469-93.

VAUGHAN, Michael. “Introduction: Henri Bergson’s Creative Evolution”, en Substance 36.3 (2007):7-24.

VIEILLARD-BARON, Jean-Louis (Ed.). Bergson: la durée et la nature. Paris: PUF, 2004.

WORMS, Frédéric. Bergson ou les deux sens de la vie. Paris: PUF, 2004.

------ Le vocabulaire de Henri Bergson. Paris: Ellipses, 2000.

YANKELEVITCH, Vladimir. Henri Bergson. Veracruz: Universidad Veracruzana, 1962. 